\section{Teaching genomics: confusion and clarity}

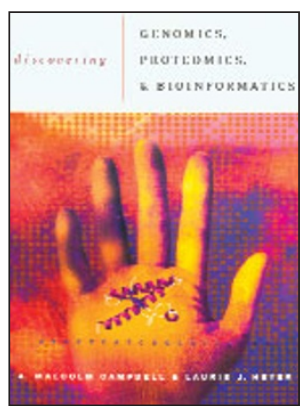

\section{Discovering Genomics, Proteomics and Bioinformatics}

by A. Malcolm Campbell \& Laurie J. Heyer

Cold Spring Harbor Laboratory Press, 2003

352 pp. paperback, $\$ 70.60$

ISBN 0-8053-4722-4

\section{Reviewed by Mark Blaxter}

Teaching genomics to undergraduate students is like vaccinating against flu: each year the novelties that have come to dominate the field have to be evaluated and course materials redesigned to prepare the student body for the ever-changing information landscape. Genomics is, at base, an attempt to show the totality of the genetics of an organism's biology, and this 'whole-picture' attitude distinguishes it from other, more reductionist approaches. Similarly, proteomics as a field is not simply the act of looking at proteins, but rather an attempt to understand the function of whole proteomes. These 'omic' approaches are not just new ways of screening an organism for a gene or gene product of interest (though that is often a very valuable outcome) but are complex, systems approaches to biology. Getting this across to students is essential and requires that they understand a wide range of basic biology and are able to deal with concepts of stochasticity and emergent behavior.

In this context, producing a genomics textbook can only be a brave attempt at hitting a moving target, but several are now available, including Brown's Genomes, Gibson and Muse's A Primer of Genome Science and the volume under review, Campbell and Heyer's Discovering Genomics, Proteomics and Bioinformatics. Each of these is very different in its focus and content. Genomes is essentially a molecular biology text with a genomic focus, whereas $A$ Primer of Genome Science is a genome's-eye view of the sorts of analyses possible with vast data sets.

Campbell and Heyer have written an integrated volume that has both didactic text, embedded 'discovery questions' for the student to answer using information in the text and available online, and a

Mark Blaxter is in the Institute of Cell, Animal and Population Biology, University of Edinburgh, UK.e-mail: mark.blaxter@ed.ac.uk linked website and CD-ROM with additional information (and many very informative color figures that, if printed, would have made the book even more expensive than it is). Some of the mathematical and statistical underpinnings are expanded on in 'Math minutes'. The book has an odd structure that suggests to this reader that it has recently been radically rearranged from an earlier teaching-tested draft.

The last three chapters are detailed and informative explorations of the roles of genomic information in issues of human medical genetics, from genetic disease to genetic pharmacology. The writing style of these chapters encourages the student to follow the development of a genomic solution to a problem but, strangely, refers to issues that "will be dealt with" in earlier chapters.

The definitions of genomics, proteomics and bioinformatics used by the authors are very partial and obscure. Molecular fingerprinting of diatoms is presented as an example of the use of 'genomic' variation (it's actually simple genetic variation), and the inclusion of insertion-mediated gene disruption and double-stranded RNA interference technologies in the chapter on 'proteomics' is contrary to their more logical presentation as gene-centered approaches. Bioinformatics, as defined by this book, is mainly the use of online databases of various sorts, the statistical analysis of microarray data and an overview of systems modeling. The core of genome sequence-based bioinformatics is ignored or assumed (for example, the second discovery question in the book assumes that the student knows how to run and interpret a BLAST search).

A welcome feature of the text is the frequent discussion of science as a discovery activity: scientists are named and located to institutions and cities, and the processes of hypothesis formulation, experimental design and testing are presented as an exciting and rewarding activity. Unfortunately for this Europe-based reader, the text annoyingly presents a US-centric world, with references to social systems that will be unfamiliar to other cultures (such as 'Title 10 money', whatever that is) and a lack of recognition of nonUS contributions to genomics. Thus, most non-US scientists are either unnamed or not located to an institution or even a country, and chief non-US initiatives, such as Ensembl (misspelled in the text and links), are sidelined.

In terms of science, there are few criticisms, though the text sometimes gets overly purple. On p 46, Campbell and Heyer write, "The unifying principle in biology is evolution, and if genomes could talk, we'd be able to hear the story of the creation of life." This trite and unscientific statement is unfortunate in the context of 'intelligent design' and 'scientific creationist' attempts to devalue evolutionary science. The book seems to have been designed for a very particular course, in a very particular college setting, and seems to translate poorly to a textbook for all. 